\title{
Use of just-about-right scales and penalty analysis to determine appropriate concentrations of stevia sweeteners for vanilla yogurt
}

\author{
P. Narayanan, ${ }^{1}$ B. Chinnasamy, L. Jin, ${ }^{2}$ and S. Clark ${ }^{3}$ \\ Department of Food Science and Human Nutrition, lowa State University, Ames 50010
}

\begin{abstract}
With the mainstream emergence of natural sweeteners such as stevia, which is available in different commercial formulations, suitability for yogurt needs to be validated. The present study aimed to determine the appropriate concentration level of 3 processed stevia sweeteners/supplements in commercial plain low-fat yogurt flavored with natural vanilla. Three different levels of sucrose, aspartame, an erythritol and $95 \%$ rebaudiana A stevia sweetener, a 95\% pure mix of maltodextrin and steviol glycosides, and a cold water stevia extract were used in the study. The just-about-right level for each sweetener and consumer acceptability of each naturally flavored low-fat vanilla yogurt were evaluated. Results from penalty analysis demonstrated that only $0.7 \%$ of stevia containing maltodextrin and $95 \%$ steviol glycoside was necessary, whereas higher levels (between 4.0 to $5.5 \%$ ) were more appropriate for stevia containing erythritol and $95 \%$ rebaudiana A or cold water extract of stevia, respectively. The concentrations of stevia sweeteners used influenced the perceived sweetness and sourness. In general, consumers disliked the yogurt sweetened with stevia or aspartame, and neither disliked nor liked the yogurt sweetened with sucrose, which was largely driven by perceived sourness of the base yogurt. The findings underline the importance of careful selection of stevia type and concentration as well as optimizing yogurt cultures and fermentation conditions before product launch.
\end{abstract}

Key words: yogurt, stevia, just-about-right scale, penalty analysis

\section{INTRODUCTION}

Probiotic dairy foods are among the predominant foods that offer health-promoting (functional) proper-

\footnotetext{
Received August 13, 2013.

Accepted January 25, 2014.

${ }^{1}$ Current address: Chobani Inc., 3450 Kimberly Road E., Twin Falls, ID 83301.

${ }^{2}$ Current address: Rutgers University, 65 Dudley Rd, New Brunswick, NJ 08901.

${ }^{3}$ Corresponding author: milkmade@iastate.edu
}

ties to the human intestinal tract (Shah, 2000; Granato et al., 2010). Among the many foods that are used as carriers to deliver probiotics, dairy foods still prove to be the most promising mode (Skovsende, 2003); cheese and yogurt appear particularly promising (Lollo et al., 2012 , 2013). Among probiotic dairy foods, yogurt is recognized as a nutrient-dense, healthy food and has gained particular popularity due to its availability as a natural flavored product containing a variety of fruits (Chandan, 2006). Besides the overall health appeal of yogurt itself, the presence of a significant amount of antioxidants (flavonoids and anthocyanin) in fruits such as strawberries, blueberries, and blackberries enhance the healthy image of yogurt (Tamime and Robinson, 1999; Chandan and O'Rell, 2006). To promote consumption of functional dairy foods by consumers with dietary restrictions, variants of "lite," low-fat, and nonfat probiotic dairy products, including yogurt, have been introduced commercially. These variants often include use of nonnutritive artificial sweeteners to improve palatability, consumer acceptability, and reduce total calories. As modern consumers seek natural foods with minimal additives, and concerns exist about additive safety, the food industry aims to create clean labels (Shim et al., 2011; Wu et al., 2012). With the awareness of plant-based high-intensity sweeteners such as stevia, processors can make "lite" products without artificial nonnutritive sweeteners.

Rebaudiana (Reb) A, isolated from Stevia rebaudiana, is relatively new to the US market as a natural sweetener. Often called sweet leaf, stevia originated in Paraguay from a genus comprising about 150 species of herbs and shrubs from the family Asteraceae. For about 1,500 yr, tribes in South America have used the leaves or extracts of stevia for its sweetening and medicinal properties. Stevia typically consists of 9 glycosides: stevioside; steviolbioside; rebaudioside A, B, C, D, E, and F; and dulcoside A (Kennelly, 2002; Starratt et al., 2002; Gardana et al., 2010). These glycosides have sweetness intensity of about 300 times that of sucrose and exhibit a high melting point and low solubility in water (Cramer and Ikan, 1987). With such stable properties, inclusion of stevia in the diet proves advantageous for diabetics and people suffering 
from obesity, heart disease, and dental caries (Kinghorn and Soejarto, 1985; Geuns, 2003). Stevia leaves have a slow onset, but long duration of sweet taste, whereas extracts often have a bitter, licorice-like aftertaste at high concentrations (Megeji et al., 2005).

Currently, stevia is available commercially in the isolated, ultrapure, crystalline form as a sweetener and in the crude form (whole or powdered leaf or as liquid extract) as a dietary supplement. Whereas the food-grade stevia sweetener is devoid of its inherent antioxidant property (i.e., antioxidant free), the dietary supplement possesses the inherent antioxidant property (i.e., it is antioxidant rich). Polyphenols present in stevia extracts inhibit hydroxyl radical, nitric oxide, superoxide anion, and hydrogen peroxide scavengers. The emerging role of antioxidants in inhibiting free radicals associated with diseases such as diabetes, cardiovascular disease, cancer, and aging (Keaney and Frei, 1994; Rice Evans, 1996; Arts and Hollman, 2005; Vita, 2005) has gained stevia attention (Ghanta et al., 2007; Shukla et al., 2009). Hence, the polyphenols in stevia might be beneficial as potential antioxidants.

Although raw stevia leaves and extracts are permitted for use as dietary supplements (McCaleb, 2006), they do not qualify for use as general sweeteners in foods due to concerns about the presence of secondary components that have pharmacological properties such as antiinflammatory, antitumor, antidiarrheal, diuretic, and immunomodulatory functions (Kinghorn, 2002). Many processes are used to purify steviol glycosides from stevia leaves. To further suppress or modulate the sweetness intensity of stevia, a variety of bulk sweeteners or fillers are added to purified stevia. Some of the fillers commonly used include sucrose, maltodextrin, lactose, inulin, and sugar alcohols (polyols) such as maltitol and erythritol. With an increasing demand for alternative sweeteners in the food industry (Haley et al., 2005), it might be beneficial to preserve the inherent antioxidant properties of stevia for use in functional food applications such as probiotic yogurt, but additional research must be conducted to evaluate suitability.

As both consumer acceptability and viability of probiotic bacteria define yogurt shelf life, the effect of natural sweeteners such as stevia on the sensory attributes and probiotic microorganisms must be investigated. Sensory attributes of yogurt with stevia sweeteners have been described using trained panelists (Guggisberg et al., 2011; Reis et al., 2011; Lisak et al., 2012), but no studies have reported consumer acceptability of yogurt with stevia. Additionally, penalty analysis has had limited application in food science publications (Endrizzi et al., 2013) and has not been reported for the sensory evaluation of yogurt. Penalty analysis, or mean drop analysis, is an emerging method in the food industry to provide direction in product development and optimization. Penalty analysis combines just-about-right (JAR) and overall liking tests to relate a decrease in consumer acceptance to attributes not at the JAR level (Lawless and Heymann, 2010). Just-about-right tests are used to measure the intensity of a specific sensory attribute (i.e., too-high, just-about-right, or too-low sweetness). Generally, a 5- or 7-point JAR scale is used to determine intensity of attributes that can affect the acceptance of the overall product. Acceptance tests are used to identify the liking of a product and individual product attributes. Rating scales for acceptance tests may be 5-, 7-, or 9-point hedonic scales, ranging from dislike extremely to like extremely (Lawless and Heymann , 2010). In the development of new products, as well as reformulating products, JAR tests are commonly used to connect consumer acceptance tests with the intensity of specific attributes to help improve consumer acceptability (Gacula et al., 2007; Villegas et al., 2010). Penalty analysis penalizes products in terms of penalty or deviation from JAR. Hence, this study was designed to investigate consumer acceptance of commercial yogurt, flavored with natural vanilla and sweetened with a variety of stevia extracts using JAR and penalty analysis.

\section{MATERIALS AND METHODS}

A commercial sample of plain low-fat yogurt (Anderson Erickson Dairy, Des Moines, IA) was used to prepare the yogurt, which was flavored with pure natural vanilla extract in 35\% alcohol (donated by Tone's Spices, Ankeny, IA). Although strawberry yogurt is more popular than vanilla yogurt, vanilla was selected because of its simplicity ( 2 ingredients). Production of strawberry yogurt would have required use of fruit pieces and building a pectin/starch based matrix gel to achieve ideal texture in final product. Ideal selection of strawberry flavoring, strawberries, sweetening, and coloring would have been more difficult and the objective was to focus on the sweeteners rather than flavoring per se. Sucrose (C\&H cane sugar; C\&H Sugar Co. Inc., Crockett, CA), aspartame (Equal; Merisant, Chicago, IL), erythritol and $95 \%$ rebaudiana A (Ery-Reb; Truvia; Cargill, Minnetonka, MN), and a 95\% pure mix of maltodextrin and steviol glycosides (Mdx-Glyc; NOW Foods, Bloomingdale, IL) were purchased from US vendors. A polyphenol-rich coldwater $(\mathbf{C W})$ extract of stevia leaves was prepared $(\mathrm{Ku}-$ towy et al., 1999) from sundried leaves of S. rebaudiana, donated by GLG Life Tech Corp. (Vancouver, Canada; origin: Qingdao, China). 


\section{Preparation of a CW Extract of Stevia Leaves (Kutowy et al., 1999)}

A 5-to- 1 ratio by weight of distilled water at $4^{\circ} \mathrm{C}$ (set at $\mathrm{pH} 2$ with food-grade phosphoric acid) and sundried leaves of $S$. rebaudiana were allowed to stand for 10 min. The supernatant was filtered through a 4-stage filter, composed of a 2-layer coffee filter, followed by a Whatman No. 4 filter paper (to remove large plant debris). The filtrate was then passed through a $0.75-\mathrm{mm}$ Nalgene polyether sulfone pore filter (to remove fine debris and plant material) and finally filtered through a $0.22-\mu \mathrm{m}$ Corning polyether sulfone pore filter (Corning Inc., Corning, NY; to remove particulates including algae, bacteria, and fungi). The extract was neutralized to $\mathrm{pH} 7$ with food-grade $\mathrm{NaOH}$ and stored below $-18^{\circ} \mathrm{C}$ until usage. All CW extracts of stevia used for the study were prepared from a single batch of stevia leaves.

\section{Determination of Steviol Glycosides in Stevia Sweeteners}

The total amount of steviol glycosides present in stevia sweeteners was determined by the ChromaDex method at ChromaDex Inc. (Irvine, CA). The analysis was based on a highly sensitive method compared with the Joint Food and Agriculture Organization of the United Nations/World Health Organization (FAO/ WHO) Expert Committee on Food Additives (JECFA) method (Hoekstra and Schaneberg, 2007). About 10 $\mu \mathrm{L}$ of each experimental sample and steviol glycoside standard (ChromaDex, Irvine, CA) was prepared with methanol and water and injected at a flow rate of 1 $\mathrm{mL} / \mathrm{min}$ at $60^{\circ} \mathrm{C}$ in a Phenomenex Synergi Hydro RP 250 reverse-phase column (Phenomenex Inc., Torrance, CA) containing Milli-Q water (Millipore Corp., Billerica, MA) and acetonitrile as the mobile phase. The total amount of steviol glycosides was detected at $202 \mathrm{~nm}$ in an Agilent 1100 HPLC system (Agilent Technologies Deutschland GmbH \& Co. KG, Waldbronn, Germany).

\section{Determination of Total Phenolics in Stevia Sweeteners and Vanilla Flavor by Folin-Ciocalteu Assay}

The total polyphenol content of triplicate samples of stevia sweeteners were determined using the Folin-Ciocalteu assay for total phenolics (Spanos and Wrolstad, 1992). Standards of gallic acid (Fisher Scientific, Pittsburgh, PA) ranging from 0.1 to $0.5 M$ were prepared. To $0.1 \mathrm{~mL}$ of sample or standard, $2 \mathrm{~mL}$ of $9 \%$ (wt/ vol) sodium carbonate solution (Fisher Scientific) and $3 \mathrm{~mL}$ of $0.2 \mathrm{~N}$ Folin-Ciocalteu reagent (Sigma-Aldrich,
St. Louis, MO) were added and mixed in a vortex for 1 min. After $2 \mathrm{~h}$, absorbances of standards and samples were measured at $765 \mathrm{~nm}$ using a Genesys 20 visible spectrophotometer (Thermo Electron Corp., Madison, WI). The total polyphenols present in the sweeteners were expressed in gallic acid equivalents (GAE) per gram of dry weight of stevia sweetener.

Results from the Folin-Ciocalteu assay were analyzed using ANOVA and pairwise comparison of treatment means using the Tukey honest significant difference test at $P=0.05$ using the statistical software JMP 8.0.2 (SAS Institute Inc., Cary, NC).

\section{Determination of Appropriate Concentration of Stevia Sweeteners for Use in Yogurt Using Sensory Analysis (JAR and Acceptance Tests)}

The objective of the study was to determine the appropriate concentration (suitable concentration of a sweetener required for the JAR level of product attributes) of stevia sweeteners and natural vanilla to add to plain commercial low-fat yogurt. In a prescreening test, samples were blindly served to experienced dairy product evaluators $(\mathrm{n}=3$; at least $10 \mathrm{~h}$ of training/experience with yogurt evaluation) at Iowa State University (Ames). The judges blindly evaluated high and low concentrations of each individual sweetener in low-fat plain yogurt, flavored with high $(2.5 \%$, wt/wt) and low $(1 \%, \mathrm{wt} / \mathrm{wt})$ levels of natural vanilla. Evaluators evaluated color, flavor, sweetness, and texture on a scale of slight, medium, and pronounced. Sweetener levels were varied due to the purity and relative sweetness of each commercial sweetener. The experienced evaluators indicated no noticeable difference in texture among the different treatments. Based upon those evaluations, high and low levels of each sweetener concentration were selected for use in consumer sensory panels (i.e., if the initial high concentration of a given sweetener was considered too high, the high level was reduced; if the initial low concentration was too low, the level was increased). It was established that the vanilla flavor needed to be increased; for subsequent consumer panels, $6 \%$ (wt/wt) vanilla was selected for flavoring.

To determine the appropriate level of vanilla and sweeteners to add to the plain yogurt, 2 consumer panels were conducted. Each 1,000-g lot of yogurt ( $\mathrm{pH} 4.7$ ) was individually blended with vanilla and sweeteners for 2 min using a hand-held manual blender and then stored in a food-grade container at $4^{\circ} \mathrm{C}$ until served to sensory panelists (within $24 \mathrm{~h}$ ). In the first panel, a lower and higher level of each sweetener was used for each yogurt $(\mathbf{C L}=$ lower and $\mathbf{C H}=$ higher concentration), with $6 \%$ (wt/wt) vanilla flavor; a total of 10 yogurt samples were available for evaluation (Table 1). In 
Table 1. Concentration of sweeteners and vanilla flavor (\%,wt/wt) for just-about-right (JAR) and hedonic acceptance tests

\begin{tabular}{|c|c|c|c|}
\hline \multirow[b]{2}{*}{ Sweetener, ${ }^{1} \%$} & \multicolumn{2}{|c|}{ Sensory test $1(\mathrm{n}=120)$} & \multirow{2}{*}{$\begin{array}{c}\text { Sensory test } 2(\mathrm{n}=60) \\
\begin{array}{c}\text { Intermediate concentration } \\
(\text { CInt; } 8.5 \% \text { vanilla })\end{array}\end{array}$} \\
\hline & $\begin{array}{l}\text { Lower concentration } \\
\text { (CL; } 6 \% \text { vanilla) }\end{array}$ & $\begin{array}{l}\text { Higher concentration } \\
\text { (CH; } 6 \% \text { vanilla) }\end{array}$ & \\
\hline Sucrose & 10.0 & 19.0 & 14.0 \\
\hline Aspartame & 1.5 & 4.0 & $1.0^{2}$ \\
\hline CW & 3.5 & 7.0 & 4.0 \\
\hline Ery-Reb & 2.5 & 6.0 & 5.5 \\
\hline Mdx-Glyc & 0.5 & 1.5 & 0.7 \\
\hline
\end{tabular}

the second panel, an intermediate level was selected for all sweeteners except aspartame (which was considered too high by panelists during the first sensory panel), and $8.5 \%$ (wt/wt) vanilla (because $6 \%$ was considered not enough flavor by panelists in panel 1), resulting in 5 yogurt samples (Table 1 ).

\section{Sensory Evaluation}

Two consumer panels [panel 1: $\mathrm{n}=120$ (40 men and 80 women); panel $2: \mathrm{n}=60$ (13 men and 47 women)], composed of volunteers aged 18 to 55, evaluated naturally flavored, low-fat vanilla yogurt. University postings were designed to recruit consumers who consume yogurt at least once per month and have no aversion to dairy products.

For the first sensory test, each consumer was seated in an individual booth in the Department of Food Science and Human Nutrition Sensory Evaluation Unit at Iowa State University, which contained a laptop computer (Dell, Plano, TX), equipped with the sensory software Compusense five (release 5.4; Compusense Inc., Guelph, Ontario, Canada). Panelists were served 4 samples (out of 10 possible yogurt samples; Table 1) in a balanced incomplete block design. In the second sensory test, each consumer was served 3 samples (out of 5 possible yogurt samples; Table 1) in a balanced incomplete block design. In both panels, not all yogurt samples were served to every consumer; however, each sample was served an equal number of times. After evaluating each sample, consumers were provided with a 2-min timed rest interval to cleanse their palates with crackers and water. In each sensory test, panelists evaluated overall acceptability of each sample and JAR levels for the product attributes. Specifically, for overall acceptability, consumers rated each sample on a 7 -point hedonic scale $(1=$ dislike very much, $2=$ dislike moderately, $3=$ dislike slightly, $4=$ neither like nor dislike, $5=$ like slightly, $6=$ like moderately, and 7
$=$ like very much). For JAR, panelists rated the same yogurt samples on a 5 -point JAR scale $(1=$ much too low, $2=$ a little too low, $3=$ just about right, $4=\mathrm{a}$ little too much, and $5=$ much too much) for color, sweetness, sourness, and vanilla flavor.

After the evaluation of all test samples, consumers were asked to complete a demographic questionnaire. Questions included age, gender, frequency of and reasons for yogurt consumption, brand of yogurt purchased, preference for a low-calorie yogurt, favorite flavor, knowledge of stevia, and willingness to purchase yogurt sweetened with stevia. Panelists had the option of "prefer not to answer" for demographic questions.

\section{Analysis of Sensory Evaluation Data by Penalty Analysis}

Penalty analysis combines JAR and overall liking tests to relate a decrease in consumer acceptance to attributes not at the JAR level (Lawless and Heymann, 2010). Generally, the preferred attributes are located at the lower left section of a plot between the mean decrease in overall liking versus percentage of not-JAR evaluations (i.e., it would have a low mean decrease in overall liking and low percentage of not-JAR evaluations). However, at non-optimal levels of an attribute, a higher mean decrease in liking and percentage of not-JAR evaluations could be observed. Sometimes, both of the not-JAR categories can receive at least $20 \%$ responses for a particular attribute. In such cases, only that not-JAR category that received the higher percentage of responses is considered for penalty analysis for that attribute. However, if both the not-JAR categories receive responses for a particular attribute, which are within $12 \%$ of each other, then each category of the not-JAR evaluations becomes a candidate for penalty analysis for that attribute. This divide in the population is known as a bipolar condition that arises due to a divide in personal preferences of consumers 
toward low or high intensities of a particular attribute (e.g., sweetness) in a product. In such cases, both the mean decrease in liking and the 2-sample $t$-test are performed separately for each category. Consequently, significant values can be obtained either for 1 or both of the not-JAR categories.

Analysis of sensory data by penalty analysis comprised the following steps (ASTM International, 2009):

1. The 5-point JAR scale was reduced to a 3-point scale consisting of 3 levels: "not enough" (by grouping "much too low" and "somewhat too low" responses), JAR, and "too much" (by grouping "much too much" and "somewhat too much" responses). The levels "not enough" and "too much" belong to the "not-JAR" category.

2 . Those attributes that received at least $20 \%$ responses (Pareto principle) in any of the 2 notJAR categories became a candidate for penalty analysis.

3. For each of the selected attributes, mean decrease in liking was calculated by subtracting the liking values obtained from the hedonic scale of the consumers in the not-JAR category from the JAR category (i.e., mean decrease $=$ JAR linking - not-JAR liking).

4. A 2-sample $t$-test was conducted between the consumers in the not-JAR and JAR categories to test for significant mean decrease in liking difference $(P<0.1)$.

5. Penalty value was calculated for those attributes that received significant mean decreases in liking through the 2-sample $t$-test, using the following formula:

$$
\begin{aligned}
\text { Penalty }= & \% \text { not-JAR } \times \text { mean decrease } \\
& \text { in overall liking, }
\end{aligned}
$$

where, $\%$ not-JAR = percentage of consumers that indicated either too much or not enough for the attribute on the JAR scale.

For penalty analysis, results from both sensory analysis tests were analyzed for significant differences using a 2-sample $t$-test $(P<0.10)$ in XL STAT 2010 software (Addinsoft, New York, NY).

\section{Statistical Analysis}

Results from overall acceptance were analyzed by ANOVA and pairwise comparison at $\alpha$ level $=0.05$, using the Tukey honest significant difference statistical feature in Compusense five.

\section{RESULTS AND DISCUSSION}

In the context of this research, the term treatment refers to stevia sweeteners (CW, Ery-Reb, and MdxGlyc) and control refers to sucrose and aspartame.

\section{Total Steviol Glycosides in Stevia Sweeteners}

Steviol glycosides are the primary sweet components present in stevia. Therefore, to determine the total percentage (wt/wt) of steviol glycoside per gram of sweetener (Table 2), a quantification method by ChromaDex HPLC (ChromaDex, Irvine, CA) was used. The commercial Mdx-Glyc had a significantly higher amount of steviol glycoside than the $\mathrm{CW}$ extract and Ery-Reb (Table 2). The results obtained correspond to anticipated results before performing the analysis; Mdx-Glyc contains fewer filler components than those present in Ery-Reb.

\section{Total Phenolics in Stevia Sweeteners and Vanilla Flavor}

Results from the current analysis of polyphenols present in stevia sweeteners (Table 2) revealed that 1 $\mathrm{g}$ of $\mathrm{CW}$ extract of stevia had $12.77 \mathrm{mg}$ of GAE of polyphenol, whereas sucrose, aspartame, and other stevia sweeteners showed negligible amounts of polyphenol activity. The natural vanilla flavoring, which was extracted from pure vanilla bean, contained about 4.61 mg of GAE/g of extract. The total polyphenol content of vanilla flavor was significantly higher than other sweeteners but lower than that of the CW extract. Results from earlier studies (Shukla et al., 2009) indicated that the crude forms of stevia contain polyphenols. This is supported by the current study. However, the study conducted by Shukla et al. (2009) was based on a hotwater extract of stevia, whereas the current study was based on a CW extract. The hot-water extract exhibited a higher amount of polyphenols ( $~ 80 \mathrm{mg}$ of GAE/g) than the $\mathrm{CW}$ extract (12.77 $\mathrm{mg}$ of $\mathrm{GAE} / \mathrm{g})$. The use of a $\mathrm{CW}$ extract enabled us to investigate a processed stevia sample that contained a significant amount of polyphenols compared with other commercial stevia sweeteners. The reason for low polyphenol activity in the commercial sweeteners (Ery-Reb and Mdx-Glyc) was likely due to the purification process used to isolate their respective steviol glycosides. Inclusion of these stevia samples in the study was to account for variability in steviol glycosides, fillers, and polyphenol content that exist in stevia sweeteners available commercially. The variability is likely due to use of numerous extraction processes adopted to modify the types of steviol 
Table 2. Concentration of total steviol glycosides and total polyphenol in stevia sweeteners and vanilla flavor expressed as gallic acid equivalents (GAE) per gram of dry weight of sweetener, and mean overall acceptance of low-fat vanilla yogurt $(n=60)$ containing selected sweeteners

\begin{tabular}{lccc}
\hline $\begin{array}{l}\text { Sweetener or vanilla } \\
\text { (concentration) }\end{array}$ & $\begin{array}{c}\text { Total steviol } \\
\text { glycosides, \% (wt/wt) }\end{array}$ & $\begin{array}{c}\text { Total polyphenol, } \\
\text { mg of GAE/g of sweetener }\end{array}$ & $\begin{array}{c}\text { Mean overall } \\
\text { acceptance }^{2}\end{array}$ \\
\hline Sucrose $(14 \%)$ & $\mathrm{NC}^{3}$ & $0.07 \pm 0.02^{\mathrm{d}}$ & $4.3^{\mathrm{A}}$ \\
Aspartame (1\%) & $\mathrm{NC}$ & $0.01 \pm 0.01^{\mathrm{d}}$ & $3.0^{\mathrm{BC}}$ \\
CW (4\%) & $0.61^{\mathrm{b}}$ & $12.77 \pm 0.06^{\mathrm{a}}$ & $2.0^{\mathrm{D}}$ \\
Ery-Reb $(5.5 \%)$ & $0.80^{\mathrm{b}}$ & $0.06 \pm 0.01^{\mathrm{d}}$ & $3.3^{\mathrm{B}}$ \\
Mdx-Glyc $(0.7 \%)$ & $15.30^{\mathrm{a}}$ & $0.06 \pm 0.00^{\mathrm{d}}$ & $2.1^{\mathrm{CD}}$ \\
Vanilla & $\mathrm{NA}^{4}$ & $4.61 \pm 0.05^{\mathrm{b}}$ & $\mathrm{NC}$ \\
\hline
\end{tabular}

${ }^{\mathrm{a}-\mathrm{d}}$ Different superscripts within a column denote significant differences $(P \leq 0.001)$.

${ }^{\mathrm{A}-\mathrm{D}}$ Different superscripts within a column denote significant differences $(P \leq 0.05)$.

${ }^{1} \mathrm{CW}=$ cold-water extract of stevia leaves; Ery-Reb $=$ erythritol and rebaudiana A; Mdx-Glyc $=$ maltodextrin and steviol glycosides.

${ }^{2}$ Seven-point scale, where $1=$ dislike very much; $4=$ neither dislike nor like; 7 = like very much.

${ }^{3} \mathrm{NC}=$ not collected. Total steviol glycosides data were not collected for sucrose or aspartame; sensory data were not collected on yogurt containing only vanilla flavor.

${ }^{4} \mathrm{NA}=$ not applicable.

glycosides present in the sweeteners. Additionally, a wide variety of bulk fillers contribute to the variability in commercial stevia sweeteners. As the use of stevia sweeteners extends to functional foods such as yogurt, it is essential to conduct studies to identify the effect of the various stevia sweeteners upon culture viability; a future manuscript will summarize the findings of our research in this area.

\section{Sensory Analysis of Yogurt and Penalty Analysis}

To determine the appropriate level of each sweetener for use in low-fat vanilla yogurt, JAR levels of the CL, $\mathrm{CH}$, and intermediate (CInt) levels of each sweetener were measured on a 5-point JAR scale. In penalty analysis, the overall acceptance and their corresponding JAR were combined to determine the significant mean decrease in consumer acceptance associated with a change from one concentration level to the other. Graphically, penalty of a particular concentration of an attribute (e.g., sweetness) is defined by its location in a dimension, defined by mean decrease in acceptance of the product on the y-axis and percentage of the population that defined the product attribute concentration as not-JAR (i.e., \% not-JAR) on the x-axis. Therefore, a product of high acceptability would possess a low mean decrease and a low percentage not-JAR. Penalty analysis uses the $20 \%$ cutoff theory on the percentage of not-JAR consumers based on the Pareto principle (i.e., the Pareto principle recognizes that " $80 \%$ of effects occur from $20 \%$ of causes" or the $80-20$ rule), and signifies several common occurrences in everyday phenomena. Therefore, the $20 \%$ cutoff is used as a general rule for penalty analysis (Klass et al., 2006; Plaehn, 2012).
The penalties corresponding to significant mean decrease in liking for each sweetener are indicated in Table 3. Generally, lower penalty values are preferred over higher penalty values when selecting samples or formulations for scale-up. The same rule of thumb was used for these studies. For all sweeteners except aspartame, CL means lower concentration, $\mathrm{CH}$ means higher concentration, and CInt means the intermediate concentration used in the second sensory panel (Table 1). If the initial high concentration of a given sweetener was deemed too high by panelists in consumer sensory test 1 , the high level was reduced for sensory test 2 ; if the initial low concentration was considered too low by panelists in consumer sensory test 1 , the level was increased for sensory test 2. Each of the concentration levels could have 2 "not-JAR" categories, namely not enough (ne) and too much (tm). Only those penalties corresponding to significant mean decrease in liking are indicated in Table 3, and were used in making recommendations for concentrations of sweeteners that should be used in commercial yogurt.

Sucrose. Because sucrose is a sweetener that most consumers are familiar with and accepting of, it was used as one control. For the sweetness attribute, all concentrations of sucrose (CL: 10\%, CH: $19 \%$, and CInt: $14 \%$ ) received a penalty by consumers (Table $3)$. The CL received the highest penalty for the sweetness attribute. Because the CInt accounted for penalty "CInt ne" and "CInt tm," CInt fell under the bipolar condition [i.e., a significant portion of the population tested considered the level CInt "not sweet enough" (ne) and a significant portion of the population thought it was "too sweet" (tm)], a less-than- $12 \%$ divide in the sweetness preferences was observed for consumers for 
NARAYANAN ET AL.

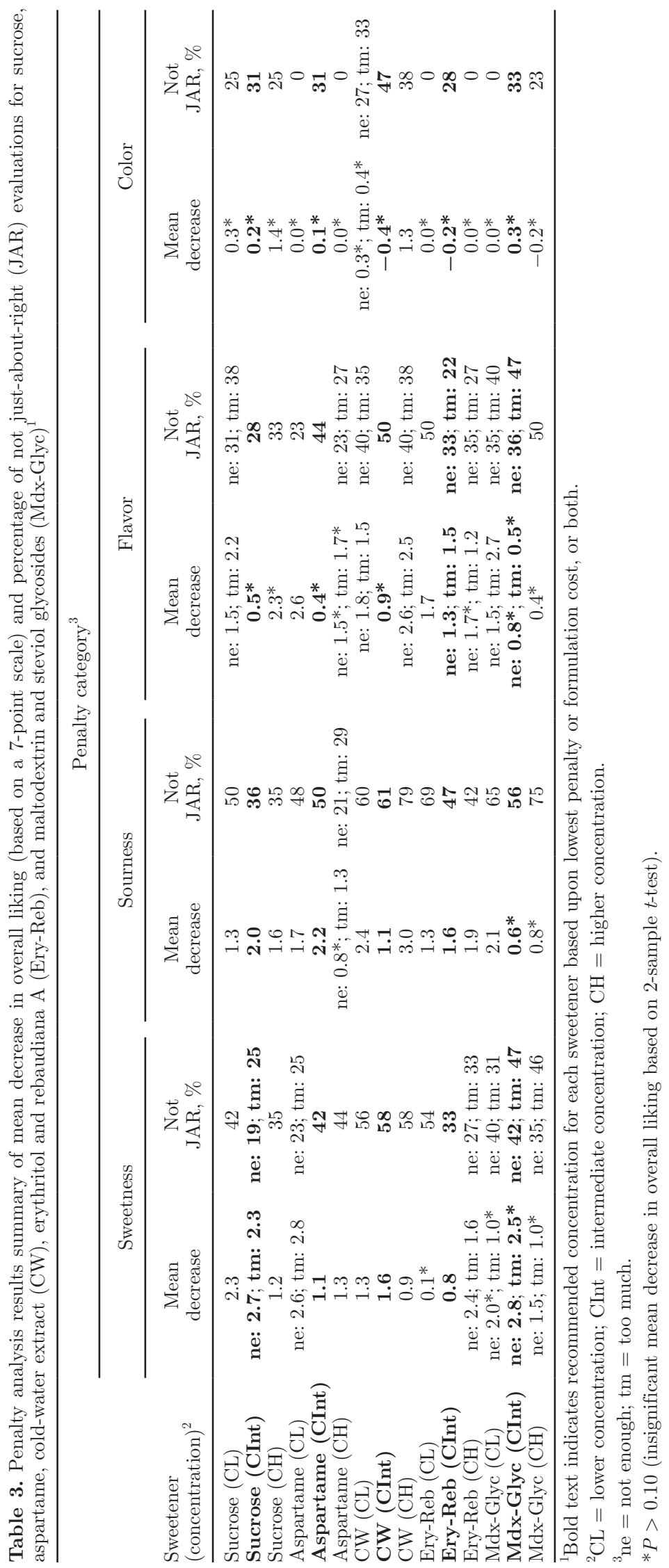


the CInt level. The penalty values for "CInt ne," "CInt tm," and $\mathrm{CH}$ had similar penalty values. However, as CInt is a lower concentration than $\mathrm{CH}$, it would be more cost-effective compared with $\mathrm{CH}$. Therefore, based upon sweetness, CInt (14\%) may be an appropriate concentration for low-fat vanilla yogurt. In other words, because penalties existed for sweetness at $\mathrm{CH}$ and CInt, these concentrations needed to be considered carefully. However, because no difference existed in the penalties of $\mathrm{CH}$ and CInt, we resorted to cost to make our recommendation.

For the sourness attribute, all sucrose concentrations experienced penalties. The CL received the highest penalty, likely because the concentration of sucrose was so low that sourness stood out to an offensive level to consumers, which led to a penalty. This research was conducted before Greek yogurt, which has a more sour profile, became popular. Although CInt and $\mathrm{CH}$ levels received higher mean decreases in overall consumer acceptance, CL received a higher penalty value because of a higher percentage of consumers that chose CL as "not-JAR."

For the flavor attribute, "CL ne" and "CL tm" accounted for penalty. As CL was the only concentration that accounted for penalty in flavor, CH $(19 \%)$ or CInt (14\%) could be potential choices of sweetener level to use for most acceptable flavor in low-fat vanilla yogurt.

Ultimately, CInt (14\%) is the most appropriate concentration of sucrose for the naturally flavored vanilla low-fat yogurt, based on no penalty in flavor, and lowest penalty for the sourness and sweetness attributes. Because overall penalty values for CInt and $\mathrm{CH}$ were comparable, we recommend the CInt level. This serves as an example of how penalty analysis can help make decisions in real world product development and formulation approach.

Aspartame. Because aspartame is a popular highintensity sweetener among food processors, it was used as a second control. Based on the sweetness attribute penalty analysis, it could be appropriate to choose the CInt $(1.0 \%)$ of aspartame for addition to yogurt (Table $3)$. On the other hand, based on sourness penalty analysis, it would be most appropriate to choose the $\mathrm{CH}(4.0 \%)$ of aspartame. As CL (1.5\%) was the only concentration that accounted for penalty in the flavor attribute, $\mathrm{CH}$ and CInt could be selected. Ultimately, CInt $(1.0 \%)$ is considered the most appropriate concentration for aspartame in vanilla yogurt because of the lower cost compared with the higher level $(\mathrm{CH})$.

$\boldsymbol{C W}$ Extract. For sweetness, all concentrations accounted for penalty. The $\mathrm{CH}(7 \%)$ received the lowest penalty, although no large differences existed among the penalty values of $\mathrm{CL}, \mathrm{CH}$, and CInt (Table 3). For the sourness attribute, the CInt (4\%) concentration of $\mathrm{CW}$ extract received the lowest penalty. Based on flavor, CInt did not account for any penalty. Based on color, CL (3.5\%) and CInt did not account for any penalty. Ultimately, CInt (4\%) is appropriate for lowfat vanilla yogurt because it accounted for the lowest penalty based on sourness, and no penalty in flavor and color.

Sweetener Ery-Reb. For the sweetness attribute, CL $(2.5 \%)$ did not account for any penalty (Table 3 ). For sourness, the CInt (5.5\%) concentration of Ery-Reb received the lowest penalty. For flavor, $\mathrm{CH}(6.0 \%)$ and CInt received lower penalty values compared with CL. In summary, CInt is recommended for Ery-Reb usage in low-fat vanilla yogurt because it accounted for the lowest penalty based on one attribute and it was the obvious choice for another attribute. Also, the CInt usage level is more cost-effective than $\mathrm{CH}$.

Sweetener Mdx-Glyc. Based on sweetness, CL $(0.5 \%)$ did not account for any penalty (Table 3 ). Based on sourness, $\mathrm{CH}(1.5 \%)$ and CInt $(0.7 \%)$ did not account for any penalty. Based on flavor, $\mathrm{CH}$ and CInt did not account for any penalty. Ultimately, CInt $(0.7 \%)$ is recommended for Mdx-Glyc use in low-fat vanilla yogurt because it is more cost-effective than $\mathrm{CH}$.

\section{Consumer Acceptance of Vanilla Yogurt with the Chosen Concentrations of Sweeteners}

Results from the demographic questions indicated that the consumer population was composed of primarily 18 - to 23 -yr-old ( $70 \%$ ) females $(79 \%)$. About $41 \%$ of consumers indicated that they consumed yogurt at least once per week (monthly yogurt consumption was listed as a requirement to participate), $87 \%$ were interested in consuming yogurt containing natural antioxidants, and $80 \%$ were interested in purchasing yogurt sweetened with stevia. Consumers indicated that fat content and type of flavor were the most influential factors in their choice of yogurt.

Results from overall consumer acceptance of yogurts with sweetener concentration CInt are summarized in Table 2. Although a consumer panel was conducted with vanilla yogurt containing concentration levels of $\mathrm{CL}$ and $\mathrm{CH}$, only results specific to concentration CInt are shown in Table 2 because CInt was estimated (through penalty analysis) to be the most appropriate concentration of sweeteners for addition in yogurt. Mean scores (2.0 to 3.3) revealed that the volunteers did not like the yogurts sweetened with stevia or aspartame. Even the yogurt with sucrose, which received the highest mean score (4.3) was neither liked nor disliked. The mean overall acceptability score for low-fat vanilla 
yogurt sweetened with sucrose was significantly higher than for those sweetened with Ery-Reb, aspartame, Mdx-Glyc, and CW extract. The results are not entirely surprising, as trained panelists in an earlier study reported bitterness and off-flavors in yogurt sweetened with stevia (Guggisberg et al., 2011). Mean scores of yogurt with Ery-Reb and of yogurt with Mdx-Glyc were not significantly different from that of yogurt with aspartame.

The error of central tendencies (Meilgaard et al., 2007) cannot be ignored, but the generally low scores for all yogurts in the present study may also be partly explained by the observation that the volunteers were not yogurt lovers; only $41 \%$ of the population reported weekly consumption of yogurt. Efforts were taken to minimize fatigue, so it is unlikely that fatigue is to blame for the low overall acceptability scores. Although samples were randomly presented, and panelists were asked to take 2 min between samples, a distasteful carryover effect from a stevia extract or the alcohol extract of natural vanilla could have lingered longer than 2 min, leading to low scores overall. Natural vanilla flavor can be a bit harsh, which is why vanillin is often used in the food industry. We selected natural vanilla because our objective was to work with all-natural ingredients (except aspartame) in this study. The use of a higher level of vanilla for panel 2 (8.5\%), compared with panel $1(6 \%)$, did not affect overall acceptability of samples (reflected by a lower mean decrease in liking for flavor at CInt compared with $\mathrm{CL}$ and $\mathrm{CH}$ ). Although not questioned in the survey, perhaps the volunteers preferred fruit yogurt rather than vanilla yogurt. The low scores could also be partly due to distaste for the base yogurt; perhaps the base yogurt itself was too sour for the panelists who participated. Though the commercially available plain yogurt was not evaluated by the volunteers, it likely influenced the overall acceptability of the samples that were manually flavored and sweetened for this project. Perhaps a yogurt with a milder flavor profile (lower titratable acidity) may be more suitable for stevia sweetening, but this hypothesis was not tested in the present work.

Although the demographic surveys indicated that most consumers (80 to $87 \%$ ) were interested in yogurt containing antioxidants or stevia, this research demonstrated that product development skill will be essential to create acceptable final products. Manufacturers must formulate and process their yogurt carefully and consistently; selecting the most suitable type and concentration of stevia and optimization of sourness will be essential to ensure acceptability and repeat purchases of yogurt sweetened with stevia.

Although similar in approach, the study conducted by Esmerino et al. (2013) on probiotic strawberry-flavored
Petit Suisse cheese differs from the current study in several aspects. The alternate sweeteners used by Esmerino et al. (2013) were laboratory-grade sweeteners, whereas our study emphasized the use of commercialgrade sweeteners in their existing form (i.e., presence of bulk filler components such as maltodextrin, and so on) to assess the overall impact that these commercial grade sweeteners can have on the sensory and microbiological profile of yogurt. Additionally, the magnitude estimation method used by Esmerino et al. (2013) was not appropriate in the present research because of the intent to know the acceptability of each attribute, rather than intensity level of each attribute.

The penalty analysis method described in the present work offers the flexibility to perform acceptance and JAR tests and compile the results to determine whether a larger percent of population indicated a small decrease in liking or a small percentage of the population indicated a large decrease in liking. Because penalty analysis can be obtained from consumer data, it requires no training beyond the regular sensory training for performing consumer acceptance and JAR tests and minimizes occurrence of errors due to ratio and scale errors commonly encountered in the magnitude estimation method.

\section{CONCLUSIONS}

Considerable variability in steviol glycosides, fillers, and polyphenol content exists in stevia sweeteners that are available commercially. As the use of stevia sweeteners extends to functional foods such as yogurt, it is essential to identify the effect of the various commercial stevia sweeteners upon yogurt quality and acceptability. Penalty analysis of 2 sensory tests, consisting of JAR ratings for attribute concentrations and overall acceptability of low-fat vanilla yogurt sweetened with various commercial stevia sweeteners revealed that intermediate sweetener levels (0.7 to $5.5 \%$, wt/wt) might be appropriate for naturally flavored vanilla lowfat yogurt. Additionally, the tests revealed that stevia sweeteners mainly influenced the perception of 2 key sensory attributes; namely, sweetness and sourness. Panelists disliked yogurt sweetened with aspartame and various commercial stevia sweeteners and neither disliked nor liked the yogurt sweetened with sucrose, which was largely driven by perceived sourness of the base yogurt. The findings underline the importance of yogurt formulation and careful selection of commercial stevia type and concentration before product launch. Adapting yogurt cultures that deliver reduced sourness in the base yogurt, use of low-fat compared with nonfat yogurt base, careful selection of type of stevia sweetener and purity, building a supporting sweetener system (us- 
ing cane sugar or mildly sweet bulk fillers such as inulin or erythritol) to prevent spikes in sweet and off-flavor notes associated with high-intensity sweeteners, and use of sweetness modulators are some of the key aspects to consider in formulating yogurt products using stevia sweeteners.

\section{ACKNOWLEDGMENTS}

The authors thank the Land O'Lakes Foundation (St. Paul, MN) for funding this research and the Product and Consumer Science team at the Kellogg Co. (Battle Creek, MI) for their technical help with penalty analysis. We sincerely appreciate Chris Fedler's support in the Sensory Evaluation Unit (Iowa State University, Ames).

\section{REFERENCES}

Arts, I. C. W., and P. C. H. Hollman. 2005. Polyphenols and disease risk in epidemiologic studies. Am. J. Clin. Nutr. 81:317S-325S.

ASTM International. 2009. Penalty Analysis or Mean Drop Analysis. MNL63-EB/MNL11493M in SEDL Manuals, Monographs and Data Series. Peryam \& Kroll Research Corp., Chicago, IL.

Chandan, R. C. 2006. Yogurt: Fruit preparations and flavoring materials. Pages 150-166 in Manufacturing Yogurt and Fermented Milks. 1st ed. R. Kevin, ed. Wiley-Blackwell Publishing, Ames, IA.

Chandan, R. C. and K. R. O'Rell. 2006. Yogurt plant: Quality assurance. Pages 247-264 in Manufacturing Yogurt and Fermented Milks. R. C. Chandan, C. H. White, A. Kilara, and Y. H. Hui, ed. Blackwell Publishing, Ames, IA.

Cramer, B., and R. Ikan. 1987. Progress in the chemistry and properties of the rebaudiosides. Developments in Sweeteners 3:45-64.

Endrizzi, I., E. Aprea, F. Biasioli, M. L. Corollaro, M. L. Demattè, M. Penasa, G. Bittante, and F. Gasperi. 2013. Implementing sensory analysis principles in the quality control of PDO products: A critical evaluation of a real-world case study. J. Sens. Stud. 28:14-24.

Esmerino, E. A., A. G. Cruz, E. P. Pereira, J. B. Rodrigues, J. A. Faria, and H. M. Bolini. 2013. The influence of sweeteners in probiotic Petit Suisse cheese in concentrations equivalent to that of sucrose. J. Dairy Sci. 96:5512-5521.

Gacula, M., Jr., S. Rutenbeck, L. Pollack, A. V. A. Resurreccion, and H. R. Moskowitz. 2007. The just-about-right intensity scale: Functional analyses and relation to hedonics. J. Sens. Stud. 22:194211.

Gardana, C., M. Scaglianti, and P. Simonetti. 2010. Evaluation of steviol and its glycosides in Stevia rebaudiana leaves and commercial sweetener by ultra-high-performance liquid chromatography-mass spectrometry. J. Chromatogr. A 1217:1463-1470.

Geuns, J. M. C. 2003. Molecules of interest: Stevioside. Phytochemistry 64:913-921.

Ghanta, S., A. Banerjee, A. Poddar, and S. Chattopadhyay. 2007. Oxidative DNA damage preventive activity and antioxidant potential of Stevia rebaudiana (Bertoni) Bertoni, a natural sweetener. J. Agric. Food Chem. 55:10962-10967.

Granato, D., G. F. Branco, A. G. Cruz, J. de Assis Fonseca Faria, and N. P. Shah. 2010. Probiotic dairy products as functional foods. Compr. Rev. Food Sci. Food Saf. 9:455-470.

Guggisberg, D., P. Piccinali, and K. Schreier. 2011. Effects of sugar substitution with Stevia, Actilight and Stevia combinations or Palatinose on rheological and sensory characteristics of low-fat and whole milk set yoghurt. Int. Dairy J. 21:636-644.

Haley, S., J. Reed, B.-H. Lin, and A. Cook. 2005. Sweetener consumption in the United States: Distribution by demographic and prod- uct characteristics. Economic Research Service Electronic Outlook Report. USDA Economic Research Service, Washington, DC.

Hoekstra, B., and B. Schaneberg. 2007. An improved HPLC method for the analysis of diterpenoid glycosides in Stevia rebaudiana. Planta Med. 73:301. (Abstr.)

Keaney, J. F., Jr., and B. Frei. 1994. Antioxidant protection of lowdensity lipoprotein and its role in the prevention of atherosclerotic vascular disease. Pages 303-351 in Natural Antioxidants in Human Health and Disease. B. Frei, ed. Academic Press, San Diego.

Kennelly, E. J. 2002. Sweet and non-sweet constituents of Stevia rebaudiana. Pages 68-85 in Stevia: The Genus Stevia. Medicinal and Aromatic Plants-Industrial Profiles. Vol. 19. A. D. Kinghorn, ed. Taylor \& Francis, London, UK.

Kinghorn, A., editor 2002. Stevia: The Genus Stevia. Medicinal and Aromatic Plants - Industrial Profiles. Vol. 19. Taylor \& Francis, London, UK.

Kinghorn, A. D., and D. D. Soejarto. 1985. Current status of stevioside as a sweetening agent for human use. Pages 1-52 in Economic and Medicinal Plant Research. Vol. 1. Academic Press, London, UK

Klass, O. S., O. Biham, M. Levy, O. Malcai, and S. Solomon. 2006. The Forbes 400 and the Pareto wealth distribution. Econ. Lett. 90:290-295.

Kutowy, O., S. Q. Zhang, and A. Kumar, inventors. 1999. Extraction of sweet compounds from Stevia rebaudiana Bertoni. National Research Council of Canada, assignee. US Pat. No. 5972120.

Lawless, H. T., and H. Heymann. 2010. Other acceptance scales and just-about right scales. Pages 328-340 in Sensory Evaluation of Food: Principles and Practices. Springer Science+Business Media LLC, New York, NY.

Lisak, K., M. Lenc, I. Jeličić, and R. Božanić. 2012. Sensory evaluation of the strawberry flavored yoghurt with stevia and sucrose addition. Croatian J. Food Technol. Biotechnol. Nutr. 7:39-43.

Lollo, P. C. B., A. G. Cruz, P. N. Morato, C. S. Moura, L. B. Carvalho-Silva, C. A. F. Oliveira, J. A. F. Faria, and J. Amaya-Farfan. 2012. Probiotic cheese attenuates exercise-induced immune suppression in Wistar rats. J. Dairy Sci. 95:3549-3558.

Lollo, P. C. B., C. S. de Moura, P. N. Morato, A. G. Cruz, W. de Freitas Castro, C. B. Betim, L. Nisishima, J. de Assis F. Faria, M. Maróstica Junior, C. O. Fernandes, and J. Amaya-Farfan. 2013. Probiotic yogurt offers higher immune-protection than probiotic whey beverage. Food Res. Int. 54:118-124.

McCaleb, R. 2006. Controversial products in the natural foods market. Herb Research Foundation. Accessed Nov. 8, 2008. http://www. herbs.org/greenpapers/controv.html.

Megeji, N. W., J. K. Kumar, V. Singh, V. K. Kaul, and P. S. Ahuja. 2005. Introducing Stevia rebaudiana, a natural zero-calorie sweetener. Curr. Sci. 88:801-804.

Meilgaard, D., G. V. Civille, and B. Carr. 2007 Sensory Evaluation Techniques. 4th ed. CRC Press, Boca Raton, FL.

Plaehn, D. 2012. CATA penalty/reward. Food Qual. Prefer. 24:141152.

Reis, R. C., V. P. R. Minim, H. M. A. Bolini, B. R. P. Dias, L. A. Minim, and E. B. Ceresino. 2011. Sweetness equivalence of different sweeteners in strawberry-flavored yogurt. J. Food Qual. 34:163-170.

Rice Evans, C. 1996. The role of antioxidants in biological systems and human studies of disease prevention. Pages 183-211 in Advances in Applied Lipid Research. F. B. Padley, ed. JAI Press, Oxford, UK.

Shah, N. P. 2000. Probiotic bacteria: Selective enumeration and survival in dairy foods. J. Dairy Sci. 83:894-907.

Shim, S.-M., S. H. Seo, Y. Lee, G.-I. Moon, M.-S. Kim, and J.-H. Park. 2011. Consumers' knowledge and safety perceptions of food additives: Evaluation on the effectiveness of transmitting information on preservatives. Food Contr. 22:1054-1060.

Shukla, S., A. Mehta, V. K. Bajpai, and S. Shukla. 2009. In vitro antioxidant activity and total phenolic content of ethanolic leaf extract of Stevia rebaudiana Bert. Food Chem. Toxicol. 47:2338-2343.

Skovsende, A. 2003. Probiotics: Good for what's bugging you. Nutraceuticals World 6:84-88. 
Spanos, G. A., and R. E. Wrolstad. 1992. Phenolics of apple, pear, and white grape juices and their changes with processing and storage. A review. J. Agric. Food Chem. 40:1478-1487.

Starratt, A. N., C. W. Kirby, R. Pocs, and J. E. Brandle. 2002. Rebaudioside F, a diterpene glycoside from Stevia rebaudiana. Phytochemistry 59:367-370.

Tamime, A. Y., and R. K. Robinson. 1999. Yoghurt: Science and Technology. Woodhead Publishing Ltd., Cambridge, UK.
Villegas, B., A. Tárrega, I. Carbonell, and E. Costell. 2010. Optimising acceptability of new prebiotic low-fat milk beverages. Food Qual. Prefer. 21:234-242.

Vita, J. A. 2005. Polyphenols and cardiovascular disease: Effects on endothelial and platelet function. Am. J. Clin. Nutr. 81:292S$297 \mathrm{~S}$.

Wu, L., Q. Zhang, L. Shan, and Z. Chen. 2012. Identifying critical factors influencing the use of additives by food enterprises in China. Food Contr. 31:425-432. 